\title{
MAKING SENSE OF THE NEW COSMOLOGY
}

\author{
Michael S. TURNER \\ Center for Cosmological Physics \\ Departments of Astronomy 68 Astrophysics and of Physics \\ Enrico Fermi Institute, The University of Chicago \\ Chicago, IL 60637-1433, USA \\ NASA/Fermilab Astrophysics Center \\ Fermi National Accelerator Laboratory \\ Batavia, IL 60510-0500, USA \\ E-mail: mturner@oddjob.uchicago.edu
}

\begin{abstract}
Over the past three years we have determined the basic features of the Universe - spatially flat; accelerating; comprised of $1 / 3$ a new form of matter, $2 / 3$ a new form of energy, with some ordinary matter and a dash of massive neutrinos; and apparently born from a burst of rapid expansion during which quantum noise was stretched to astrophysical size seeding cosmic structure. The New Cosmology greatly extends the highly successful hot big-bang model. Now we have to make sense of all this: What is the dark matter particle? What is the nature of the dark energy? Why this mixture? How did the matter - antimatter asymmetry arise? What is the underlying cause of inflation (if it indeed occurred)?
\end{abstract}

\section{The New Cosmology}

Cosmology is enjoying the most exciting period of discovery yet. Over the past three years a new, improved standard cosmology has been emerging. It incorporates the highly successful standard hot big-bang cosmology' and extends our understanding of the Universe to times as early as $10^{-32} \mathrm{sec}$, when the largest structures in the Universe were still subatomic quantum fluctuations.

This New Cosmology is characterized by

- Flat, critical density accelerating Universe

- Early period of rapid expansion (inflation)

- Density inhomogeneities produced from quantum fluctuations during inflation

- Composition: $2 / 3$ dark energy; $1 / 3$ dark matter; $1 / 200$ bright stars

- Matter content: $(29 \pm 4) \%$ cold dark matter; $(4 \pm 1) \%$ baryons; $\gtrsim 0.3 \%$ neutrinos

- Current temperature: $T=2.725 \pm 0.001 \mathrm{~K}$ 
- Current age: $14 \pm 1$ Gyr

- Current expansion rate: $72 \pm 7 \mathrm{~km} \mathrm{~s}^{-1} \mathrm{Mpc}^{-1}$

The New Cosmology is certainly not as well established as the standard hot big bang. However, the evidence is mounting.

\subsection{Mounting Evidence: Recent Results}

The position of the first acoustic peak in the multipole power spectrum of the anisotropy of the cosmic microwave background (CMB) radiation provides a powerful means of determining the global curvature of the Universe. With the recent DASI observations of CMB anisotropy on scales of one degree and smaller, the evidence that the Universe is at most very slightly curved is quite firm. The curvature radius of the Universe $\left(\equiv R_{\text {curv }}\right)$ and the total energy density parameter $\Omega_{0}=\rho_{\mathrm{TOT}} / \rho_{\text {crit }}$, are related:

$$
R_{\text {curv }}=H_{0}^{-1} /\left|\Omega_{0}-1\right|^{1 / 2}
$$

The spatial flatness can be expressed as $\Omega_{0}=1.0 \pm 0.04$, or said in words, the curvature radius is at least 50 times greater than the Hubble radius.

As I will discuss in great detail later, the evidence for accelerated expansion is also very strong.

The series of acoustic peaks in the CMB multipole power spectrum and their heights indicate a nearly scale-invariant spectrum of adiabatic density perturbations with $n=1 \pm 0.07$. Nearly scale-invariant density perturbations and a flat Universe are two of the three hallmarks of inflation. Thus, we are beginning to see the first significant experimental evidence for inflation, the driving idea in cosmology for the past two decades.

The striking agreement of the BBN determination of the baryon density from measurements of the primeval deuterium abundance, $\Omega_{B} h^{2}=0.020 \pm$ 0.001 , with those from from recent CMB anisotropy measurements, $\Omega_{B} \Omega^{2}=$ $0.022 \pm 0.004$, make a strong case for a small baryon density, as well as the consistency of the standard cosmology. (Here, $h=H_{0} / 100 \mathrm{~km} \mathrm{sec}^{-1} \mathrm{Mpc}^{-1}$.) There can now be little doubt that baryons account for but a few percent of the critical density.

Our knowledge of the total matter density is improving, and becoming less linked to the distribution of light. This makes determinations of the matter less sensitive to the uncertain relationship between the clustering of mass and of light (what astronomers call the bias factor). 5 Both the CMB and clusters of galaxies allow a determination of the ratio of the total matter density (anything that clusters - baryons, neutrinos, cold dark matter) to that in ordinary matter: 
$\Omega_{M} / \Omega_{B}=7.2 \pm 2.1$ (CMB) and $=9 \pm 1.5$ (clusters). Not only are these numbers consistent, they make a very strong case for something beyond quark-based matter. When combined with our knowledge of the baryon density, one infers a total matter density of $\Omega_{M}=0.33 \pm 0.04 .$.

The many successes of the cold dark matter (CDM) scenario - from the sequence of structure formation (galaxies first, clusters of galaxies and larger objects later) and the structure of the intergalactic medium, to its ability to reproduce the power spectrum of inhomogeneity measured today - makes it clear that CDM holds much, if not all, of the truth in describing the formation of structure in the Universe. This implies that whatever the dark matter particle is, it moves slowly (i.e., the bulk of the matter cannot be in the form of hot dark matter such as neutrinos) and interacts only weakly (e.g., with strength much less than electromagnetic) with ordinary matter.

The evidence from SuperKamiokande- for neutrino oscillations makes a strong case that neutrinos have mass $\left(\sum_{i} m_{\nu} \gtrsim 0.1 \mathrm{eV}\right)$ and therefore contribute to the mass budget of the Universe at a level comparable to or greater than that of bright stars. Particle dark matter has moved from the realm of a hypothesis to a quantitative question - how much of each type of particle dark matter. Structure formation in the Universe (especially the existence of small scale structure)-suggests that neutrinos contribute at most $5 \%$ or $10 \%$ of the critical density.

Even the age of the Universe and the pesky Hubble constant have been reined in. The uncertainties in the ages of the oldest globular clusters have been better identified and quantified, leading to a more precise age, $t_{0}=13.5 \pm$ 1.5 Gyr.. The CMB can be used to constrain the expansion age, independent of direct $t_{1}$ measurements of $H_{0}$ or the composition of the Universe, $t_{\exp }=14 \pm$ 0.5 Gyr.- A host of different techniques are consistent with the Hubble constant determined by the HST key project, $H_{0}=72 \pm 7 \mathrm{~km} \mathrm{~s}^{-1} \mathrm{M}_{\mathrm{Pc}}{ }^{-1}$. Further, the error budget is now well understood and well quantified.10! Moreover, the expansion age derived from this Hubble, which depends upon the composition of the Universe, is consistent with the previous two ages.

The poster child for precision cosmology continues to be the present temperature of the CMB. It wass-determined by the FIRAS instrument on COBE to be: $T_{0}=2.725 \pm 0.001 \mathrm{~K} .1 .1$.

\subsection{Successes and Consistency Tests}

To sum up; we have determined the basic features of the Universe: the cosmic matter/energy budget; a self consistent set of cosmological parameters with

realistic errors; and the global curvature. Two of the three key predictions of 
inflation - flatness and nearly scale-invariant, adiabatic density perturbations - have passed their first significant tests. Last but not least, the growing quantity of precision data are now testing the consistency of the FriedmannRobertson-Walker framework and General Relativity itself.

In particular, the equality of the baryon densities determined from BBN and CMB anisotropy is remarkable. The first involves nuclear physics when the Universe was seconds old, while the latter involves gravitational and classical electrodynamics when the Universe was 400,000 years old.

The entire framework has been tested by the existence of the aforementioned acoustic peaks in the CMB angular power spectrum. They reveal largescale motions that have remained coherent over hundreds of thousands of years, through a delicate interplay of gravitational and electromagnetic interactions.

Another test of the basic framework is the accounting of the density of matter and energy in the Universe. The CMB measurement of spatial flatness implies that the matter and energy densities must sum to the critical density. Measurements of the matter density indicate $\Omega_{M}=0.33 \pm 0.04$; and measurements of the acceleration of the Universe from supernovae indicate the existence of a smooth dark energy component that accounts for $\Omega_{X} \sim 0.67$ (depending upon the equation of state).

Finally, while cosmology has in the past been plagued by "age crises" time back to the big bang (expansion age) apparently less than the ages of the oldest objects within the Universe - today the ages determined by very different and independent techniques point to a consistent age of $14 \mathrm{Gyr}$.

\section{Cosmic Mysteries}

Cosmological measurements and observations over the next decade will test (and probably refine) the New Cosmology. 12 If we are fortunate, they will also help us to make sense of it. At the moment, there are a number of cosmic mysteries, which provide opportunities for surprises and new insights. Here I will quickly go through my list, and save the most intriguing to me - dark energy - for its own section at the end.

\subsection{Dark Matter}

By now, the conservative hypothesis is that the dark matter consists of a new form of matter, with the axion and neutralino as the leading candidates. That most of the matter in the Universe exists in a new form of matter - yet detected in the laboratory - is a bold and as of yet untested assertion.

Experiments to directly detect the neutralinos or axions holding our own galaxy together have now reached sufficient sensitivity to probe the regions 
of parameter space preferred by theory. In addition, the neutralino can be created by upcoming collider experiments (at the Tevatron or the LHC), or detected by its annihilation signatures.13i

While the CDM scenario is very successful there are some nagging problems. They may point to a fundamental difficulty or may be explained by messy astrophysics.14 The most well known of these problems are the prediction of cuspy dark matter halos (density profile $\rho_{\mathrm{DM}} \rightarrow 1 / r^{n}$ as $r \rightarrow 0$, with $n \simeq 1-1.5$ ) and the apparent prediction of too much substructure. While there are plausible astrophysical explanations for both problems, they could indicate an unexpected property of the dark matter particle (e.g., large self interaction cross section! 15 or annihilation cross section- $\left.{ }_{-}^{11}\right)$ or perhaps even a failure of the particle dark matter paradigm.

I leave for the "astrophysics to do list" an accounting of the dark baryons. Since $\Omega_{B} \simeq 0.04$ and $\Omega_{*} \simeq 0.005$, the bulk of the baryons are optically dark. In clusters, the dark baryons have been identified: they exists as hot, x-ray emitting gas. Elsewhere, the dark baryons have not been identified. According to CDM, the bulk of the dark baryons are likely to exist in hot/warm gas associated with galaxies, but this gas has not been detected. Since clusters account for only about 5 percent of the total mass, the bulk of the dark baryons are still not accounted for.

\subsection{Baryogenesis}

The origin of ordinary, quark-based matter is not yet fully understood. If the Universe underwent inflation, the dynamical, post-inflation production of an excess of baryons over antibaryons - baryogenesis - is obligatory, since any pre-inflation baryon asymmetry is diluted away by the enormous entropy production associated with reheating.

Because we now know that electroweak processes violate $B+L$ at a very rapid rate at temperatures above $100 \mathrm{GeV}$ or so, baryogenesis is more constrained than when the idea was introduced more than twenty years ago. Today there are three possibilities: 1) produce the baryon asymmetry by GUT-scale physics with $B-L \neq 0$ (to prevent it being subsequently washed away by $B+L$ violation); 2) produce a lepton asymmetry $(L \neq 0)$, which is then transmuted into the baryon asymmetry by electroweak $B+L$ violation; or 3 ) produce the baryon asymmetry during the electroweak phase transition using electroweak $B$ violation.

While none of the three possibilities can be ruled out, the second possibility looks most promising, and it adds a new twist to the origin of quark-based matter: We are here because neutrinos have mass. In the lepton asymmetry 
first scenario, Majorana neutrino mass provides the requisite lepton number violation. The drawback of the first possibility is the necessity of a high reheat temperature after inflation, $T_{\mathrm{RH}} \gg 10^{5} \mathrm{GeV}$, which is difficult to achieve in most models of inflation. The last possibility, while very attractive because all the input physics might be measurable at accelerator, requires new sources of $C P$ violation at $\mathrm{TeV}$ energies as well as a strongly first-order electroweak phase transition.

\subsection{Inflation}

There are still many questions to be answered about inflation, including the most fundamental: did inflation (or something similar) actually take place!

A good program is in place to test the inflationary framework. This is done by testing its three basic predictions: spatially flat Universe; nearly-scale invariant, nearly power-law spectrum of Gaussian adiabatic, density perturbations; and a spectrum of nearly scale-invariant gravitational waves.

The first two predictions will be probed much more sharply over the next decade. The value of $\Omega_{0}$ should be determined to much better than 1 percent. The spectral index $n$ that characterizes the density perturbations should be measured to percent accuracy.

Generically, inflation predicts $|n-1| \sim \mathcal{O}(0.1)$, where $n=1$ corresponds to exact scale invariance. Likewise, the deviations from an exact power-law predicted by inflation, $1-17 \ln |d n / d \ln k| \sim 10^{-4}-10^{-2}$ will be tested. The CMB and the abundance of rare objects such as clusters of galaxies will allow the prediction of Gaussianity to be tested.

Inflationary theory has given little guidance as to the amplitude of the gravitational waves produced during inflation. If detected, they are a smokin' gun prediction. Their amplitude is directly related to the scale of inflation, $h_{\mathrm{GW}} \simeq H_{\text {inflation }} / m_{\mathrm{Pl}}$. Together measurements of $n-1$ and $d n / \ln k$, they can reveal much about the underlying scalar potential driving inflation. Measuring their spectral index - a most difficult łask - provides a consistency test of the single scalar-field model of inflation.1 1 -

\subsection{The Dimensionality of Space-time}

Are there additional spatial dimensions beyond the three for which we have very firm evidence? I cannot think of a deeper question in physics today. If there are new dimensions, they are likely to be relevant for cosmology, or at

least raise new questions in cosmology (e.g., why are only three dimensions large? what is going on in the bulk? and so on). Further, cosmology may well be the best means for establishing the existence of extra dimensions. 


\subsection{Before Inflation, Other Big-bang Debris, and Surprises}

Only knowing everything there is to know about the Universe would be worse than knowing all the questions to ask about it. Without doubt, as our understanding deepens, new questions and new surprises will spring forth.

The cosmological attraction of inflation is its ability to make the present state of the Universe insensitive to its initial state. However, should we establish inflation as part of cosmic history, I am certain that cosmologists will begin asking what happened before inflation.

Progress in cosmology depends upon studying relics. We have made much of the handful we have - the light elements, the baryon asymmetry, dark matter, and the CMB. The significance of a new relic cannot be overstated. For example, detection of the cosmic sea of neutrinos would reveal the Universe at 1 second. Identifying the neutralino as the dark matter particle and determining its properties at an accelerator laboratory would open a window on the Universe at $10^{-8} \mathrm{sec}$. By comparing its relic abundance as derived from its mass and cross section with its actual abundance measured in the Universe, one could test cosmology at the time the neutralino abundance was determined.

And then there are relics that cosmologists have yet to dream of!

\section{Dark Energy: Seven Lessons}

The dark energy accounts for $2 / 3$ of the stuff in the Universe and determines its destiny. That puts it high on the list of outstanding problems in cosmology. Its deep connections to fundamental physics - a new form of energy with repulsive gravity and possible implications for the divergences of quantum theory and supersymmetry breaking - put it very high on the list of outstanding problems in particle physics.

Dark energy is my term for the causative agent of the current epoch of accelerated expansion. According to the second Friedmann equation,

$$
\frac{\ddot{R}}{R}=-\frac{4 \pi G}{3}(\rho+3 p)
$$

this stuff must have negative pressure, with magnitude comparable to its energy density, in order to produce accelerated expansion [recall $q=-(\ddot{R} / R) / H^{2} ; R$ is the cosmic scale factor]. Further, since this mysterious stuff does not show its presence in galaxies and clusters of galaxies, it must be relatively smoothly distributed.

That being said, dark energy has the following defining properties: (1) it emits no light; (2) it has large, negative pressure, $p_{X} \sim-\rho_{X}$; and (3) it is approximately homogeneous (more precisely, does not cluster significantly 
with matter on scales at least as large as clusters of galaxies). Because its pressure is comparable in magnitude to its energy density, it is more "energylike" than "matter-like" (matter being characterized by $p \ll \rho$ ). Dark energy is qualitatively very different from dark matter, and is certainly not a replacement for it.

It has been said that the sum total of progress in understanding the acceleration of the Universe is naming the causative agent. While not too far from the truth, there has been some progress. I will summarize it below.

\subsection{Two Lines of Evidence for an Accelerating Universe}

Two lines of evidence point to an accelerating Universe. The first is the direct evidence based upon measurements of type la supernovae carried out by two, groups, the Supernova Cosmology Project 19 and the High- $z$ Supernova Team.20 These two teams used different analysis techniques and different samples of high- $z$ supernovae and came to the same conclusion: the expansion of the Universe is speeding up, not slowing down.

The recent sęęndipitous discovery of a supernovae at $z=1.76$ bolsters the case significantly $y_{-1}^{211}$ and provides the first evidence for an early epoch of decelerated expansion.22 $\mathrm{SN} 1997 \mathrm{ff}$ falls right on the accelerating Universe curve on the magnitude - redshift diagram, and is a magnitude brighter than expected in a dusty open Universe or an open Universe in which type Ia supernovae are systematically fainter at high- $z$.

The second, independent line of evidence for the accelerating Universe comes from measurements of the composition of the Universe, which point to a missing energy component with negative pressure. The argument goes like this: CMB anisotropy measurements indicate that the Universe is nearly flat, with density parameter, $\Omega_{0}=1.0 \pm 0.04$. In a flat Universe, the matter density and energy density must sum to the critical density. However, matter only contributes about $1 / 3$ of the critical density, $\Omega_{M}=0.33 \pm 0.04$. (This is based upon measurements of CMB anisotropy, of bulk flows, and of the baryonic fraction in clusters.) Thus, two thirds of the critical density is missing! Doing the bookkeeping more precisely, $\Omega_{X}=0.67 \pm 0.06$.

In order to have escaped detection, this missing energy must be smoothly distributed. In order not to interfere with the formation of structure (by inhibiting the growth of density perturbations), the energy density in this component must change more slowly than matter (so that it was subdominant in the past). For example, if the missing $2 / 3$ of critical density were smoothly distributed matter $(p=0)$, then linear density perturbations would grow as $R^{1 / 2}$ rather than as $R$. The shortfall in growth since last scattering $(z \simeq 1100)$ 
would be a factor of 30 , far too little growth to produce the structure seen today.

The pressure associated with the missing energy component determines how it evolves:

$$
\begin{array}{r}
\rho_{X} \propto R^{-3(1+w)} \\
\rho_{X} / \rho_{M} \propto(1+z)^{3 w}
\end{array}
$$

where $w$ is the ratio of the pressure of the missing energy component to its energy density (here assumed to be constant). Note, the more negative $w$, the faster the ratio of missing energy to matter goes to zero in the past. In order to grow the structure observed today from the density perturbations indicated hy CMB anisotropy measurements, $w$ must be more negative than about $-\frac{1}{2}$.

For a flat Universe the deceleration parameter today is

$$
q_{0}=\frac{1}{2}+\frac{3}{2} w \Omega_{X} \sim \frac{1}{2}+w
$$

Therefore, knowing $w<-\frac{1}{2}$ implies $q_{0}<0$ and accelerated expansion. This independent argument for accelerated expansion and dark energy makes the supernova case all the more compelling.

\subsection{Gravity Can Be Repulsive in Einstein's Theory, But...}

In Newton's theory, mass is the source of the gravitational field and gravity is always attractive. In General Relativity, both energy and pressure source the gravitational field. This fact is reflected in Eq. 11.'. Sufficiently large negative pressure leads to repulsive gravity.

While accelerated expansion can be accommodated within Einstein's theory, that does not preclude that the ultimate explanation lies in a fundamental modification of Einstein's theory. Lacking any good ideas for such a modification, I will discuss how accelerated expansion fits in the context of General Relativity. If the explanation for the accelerating Universe ultimately fits within the Einsteinian framework, it will be a stunning new triumph for General Relativity.

\subsection{The Biggest Embarrassment in all of Theoretical Physics}

Einstein introduced the cosmological constant to balance the attractive gravity of matter. He quickly discarded the cosmological constant after the discovery of the expansion of the Universe. Whether or not Einstein appreciated that his theory predicted the possibility of repulsive gravity is unclear to me. 
The advent of quantum field theory made consideration of the cosmological constant obligatory not optional: The only possible covariant form for the energy of the (quantum) vacuum,

$$
T_{\mathrm{VAC}}^{\mu \nu}=\rho_{\mathrm{VAC}} g^{\mu \nu},
$$

is mathematically equivalent to the cosmological constant. It takes the form for a perfect fluid with energy density $\rho_{\mathrm{VAC}}$ and isotropic pressure $p_{\mathrm{VAC}}=-\rho_{\mathrm{VAC}}$ (i.e., $w=-1$ ) and is precisely spatially uniform. Vacuum energy is almost the perfect candidate for dark energy.

Here is the rub: the contributions of well-understood physics (say up to the $100 \mathrm{GeV}$ scale) to the quantum-vacuum energy add up to $10^{55}$ times the present critical density. (Put another way, if this were so, the Hubble time would be $10^{-10} \mathrm{sec}$, and the associated event hprizon would be $3 \mathrm{~cm}$ !) This is the well known cosmological-constant problem.24

While string theory currently offers the best hope for marrying gravity to quantum mechanics, it has shed precious little light on the cosmological constant problem, other than to speak to its importance. Thomas has suggested that using the holographic principle to count the available number of states in our Hubble volume leads to an upper bound on the vas,uum energy that is comparable to the energy density in matter + radiation.26 While this reduces the magnitude of the cosmological-constant problem very significantly, it does not solve the dark energy problem: a vacuum energy that is always comparable to the matter + radiation energy density would strongly suppress the growth of structure.

The deSitter space associated with the accelerating Universe may pose serious problems for the formulation of string theory!2? Banks and Dine argue that all explanations for dark energy suggested thus far are incompatible with perturbative string theory.2 At the very least there is high tension between accelerated expansion and string theory.

The cosmological constant problem leads to a fork in the dark-energy road: one path is to wait for theorists to get the "right answer" (i.e., $\Omega_{X}=2 / 3$ ); the other path is to assume that even quantum nothingness weighs nothing and something else with negative pressure must be causing the Universe to speed up. Of course, theorists follow the advice of Yogi Berra: "When you see a fork in the road, take it."

\subsection{Parameterizing Dark Energy: For Now, It's w}

Theorists have been very busy suggesting all kinds of interesting possibilities for the dark energy: networks of topological defects, rolling or spinning scalar fields 
(quintessence and spintessence), influence of "the bulk", and the breakdown of the Friedmann equations.25 2 . An intriguing recent paper suggests dark matter and dark energy are connected through axion physics.

In the absence of compelling theoretical guidance there is a simple way to parameterize dark energy, by its equation-of-state $w .231$

The uniformity of the CMB testifies to the near isotropy and homogeneity of the Universe. This implies that the stress-energy tensor for the Universe must take the perfect fluid form. . $^{\prime}$ Since dark energy dominates the energy budget, its stress-energy tensor must, to a good approximation, take the form

$$
T_{X}^{\mu} \approx \operatorname{diag}\left[\rho_{X},-p_{X},-p_{X},-p_{X}\right]
$$

where $p_{X}$ is the isotropic pressure and the desired dark energy density is

$$
\rho_{X}=2.7 \times 10^{-47} \mathrm{GeV}^{4}
$$

(for $h=0.72$ and $\Omega_{X}=0.66$ ). This corresponds to a tiny energy scale, $\rho_{X}^{1 / 4}=2.3 \times 10^{-3} \mathrm{eV}$.

The pressure can be characterized by its ratio to the energy density (or equation-of-state):

$$
w \equiv p_{X} / \rho_{X}
$$

Note, $w$ need not be constant; e.g., it could be a function of $\rho_{X}$ or an explicit function of time or redshift. ( $w$ can always be rewritten as an implicit function of redshift.)

For vacuum energy $w=-1$; for a network of topological defects $w=-N / 3$ where $N$ is the dimensionality of the defects ( 1 for strings, 2 for walls, etc.). For a minimally coupled, rolling scalar field,

$$
w=\frac{\frac{1}{2} \dot{\phi}^{2}-V(\phi)}{\frac{1}{2} \dot{\phi}^{2}+V(\phi)}
$$

which is time dependent and can vary between -1 (when potential energy dominates) and +1 (when kinetic energy dominates). Here $V(\phi)$ is the potential for the scalar field.

I believe that for the foreseeable future getting at the dark energy will mean trying to measure its equation-of-state, $w(t)$.

\subsection{The Universe: The Lab for Studying Dark Energy}

Dark energy by its very nature is diffuse and a low-energy phenomenon. It probably cannot be produced at accelerators; it isn't found in galaxies or even 
clusters of galaxies. The Universe itself is the natural lab - perhaps the only lab - in which to study it.

The primary effect of dark energy on the Universe is on the expansion rate. The first Friedmann equation can be written as

$$
H^{2}(z) / H_{0}^{2}=\Omega_{M}(1+z)^{3}+\Omega_{X} \exp \left[3 \int_{0}^{z}[1+w(x)] d \ln (1+x)\right]
$$

where $\Omega_{M}\left(\Omega_{X}\right)$ is the fraction of critical density contributed by matter (dark energy) today, flatness is assumed, and the dark-energy term follows from energy conservation, $d\left(\rho_{X} R^{3}\right)=-p_{X} d R^{3}$. For constant $w$ the dark energy term is simply $\Omega_{X}(1+z)^{3(1+w)}$. Note, with the assumption of flatness, $H(z) / H_{0}$ depends upon only two parameters: $\Omega_{M}$ and $w(z)$.

While $H(z)$ is probably not directly measurable (however see Loebes ${ }^{3}$ ), it does affect two observable quantities: the (comoving) distance to an object at redshift $z$,

$$
r(z)=\int_{0}^{z} \frac{d z}{H(z)}
$$

and the growth of (linear) density perturbations, governed by

$$
\ddot{\delta}_{k}+2 H \dot{\delta}_{k}-4 \pi G \rho_{M} \delta_{k}=0
$$

where $\delta_{k}$ is the Fourier component of comoving wavenumber $k$ and overdot indicates $d / d t$.

The comoving distance $r(z)$ can be probed by standard candles (e.g., type Ia supernovae) through the classic cosmological observable, luminosity distance $d_{L}(z)=(1+z) r(z)$. It can also be probed by counting objects of a known intrinsic comoving number density, through the comoving volume element, $d V / d z d \Omega=r^{2}(z) / H(z)$.

Both galaxies and clusters of galaxies have been suggested as objects to count. 12 For each, their comoving number density evolves (in the case of clusters very significantly). However, it is believed that much, if not all, of the evolution can be modelled through numerical simulations and semi-analytical calculations in the CDM picture. In the case of clusters, evolution is so significant that the number count test probe is affected by dark energy through both $r(z)$ and the growth of perturbations, with the latter being the dominant effect.

The various cosmological approaches to-ferreting out the nature of the dark energy have been, studied extensively. 33 - Based largely upon my work with Dragan Huterer, 34 I I summarize what we know about the efficacy of the cosmological probes of dark energy: 
- Present cosmological observations prefer $w=-1$, with a $95 \%$ confidence limit $w<-0.6 .36$.

- Because dark energy was less important in the past, $\rho_{X} / \rho_{M} \propto(1+$ $z)^{3 w} \rightarrow 0$ as $z \rightarrow \infty$, and the Hubble flow at low redshift is insensitive to the composition of the Universe, the, most sensitive redshift interval for probing dark energy is $z=0.2-2.34$

- The CMB has limited power to probe $w$ (e.g., the projected precision for Planck is $\left.\sigma_{w}=0.25\right)$ and no power to probe its time variation ? ? $^{34}$

- A high-quality sample of $2000 \mathrm{SNe}$ distributed from $z=0.2$ to $z=$ 1.7 could measure $w$ to a precision $\sigma_{w}=0.05$ (assuming an irreducible systematic error of $0.14 \mathrm{mag}$ ). If $\Omega_{M}$ is known independently to better than $\sigma_{\Omega_{M}}=0.03, \sigma_{w}$ improves by a factor of three and the, rate of change of $w^{\prime}=d w / d z$ can be measured to precision $\sigma_{w^{\prime}}=0.16$.

- Counts of galaxies and of clusters of galaxies may have the same potential to probe $w$ as SNe Ia. The critical issue is systematics (including the evolution of the intrinsic comoving number density, and the ability to identify galaxies or clusters of a fixed mass).321.

- Measuring weak gravitational lensing by large-scale structure over a field of 1000 square degrees (or more) could have comparable sensitivity to $w$ as type Ia supernovae. However, weak gravitational lensing does not appear to be a good method to probe the time variation of $w . l^{35}$. The systematics associated with weak gravitational lensing have not yet been studied carefully and could limit its potential.

- Some methods do not look promising in their ability to probe $w$ because of irreducible systematics (e.g., Alcock - Paczynski test and strong gravitational lensing of QSOs). However, both could provide important independent confirmation of accelerated expansion.

\subsection{Why now?: The Nancy Kerrigan Problem}

A critical constraint on dark energy is that it not interfere with the formation of structure in the Universe. This means that dark energy must have been relatively unimportant in the past (at least back to the time of last scattering, $z \sim 1100$ ). If dark energy is characterized by constant $w$, not interfering with structure formation can be quantified as: $w \lesssim-\frac{1}{2} \cdot 23$ This means that the 
dark-energy density evolves more slowly than $R^{-3 / 2}$ (compared to $R^{-3}$ for matter) and implies

$$
\begin{array}{ll}
\rho_{X} / \rho_{M} \rightarrow 0 & \text { for } t \rightarrow 0 \\
\rho_{X} / \rho_{M} \rightarrow \infty & \text { for } t \rightarrow \infty
\end{array}
$$

That is, in the past dark energy was unimportant and in the future it will be dominant! We just happen to live at the time when dark matter and dark energy have comparable densities. In the words of Olympic skater Nancy Kerrigan, "Why me? Why now?"

Perhaps this fact is an important clue to unraveling the nature of the dark energy. Perhaps not. I shudder to say this, but it could be at the root of an anthropic explanation for the size of the cosmological constant: The cosmological constant is as large as it can be and still allow the formation of structures that can support life?

\subsection{Dark Energy and Destiny}

Almost everyone is aware of the connection between the shape of the Universe and its destiny: positively curved recollapses, flat; negatively curved expand forever. The link between geometry and destiny depends upon a critical assumption: that matter dominates the energy budget (more precisely, that all components of matter/energy have equation of state $w>-\frac{1}{3}$ ). Dark energy does not satisfy this condition.

In a Universe with dark energy the connection between geometry and destiny is severed. 18.4 A flat Universe (like ours) can continue expanding exponentially forever with the number of visible galaxies diminishing to a few hundred (e.g., if the dark energy is a true cosmological constant); the expansion can slow to that of a matter-dominated model (e.g., if the dark energy dissipates and becomes sub dominant); or, it is even possible for the Universe to recollapse (e.g., if the dark energy decays revealing a negative cosmological constant). Because string theory prefers anti-deSitter space, the third possibility should not be forgotten.

Dark energy then is the key to understanding our destiny.

\subsection{The Challenge}

I believe the really big challenge for the New Cosmology is making sense of dark energy. 
Because of its diffuse character, the Universe is likely the lab where dark energy can best be attacked (though one should not rule other approaches e.g., if the dark energy_involves a ultra-light scalar field, then there should be a new long-range force 39 ).

While type Ia supernovae look particularly promising - they have a track record and can, in principle, be used to map out $r(z)$ - there are important open issues. Are they really standardizable candles? Have they evolved? Is the high-redshift population the same as the low-redshift population?

The dark-energy problem is important enough that pursuing complimentary approaches is both justified and prudent. Weak-gravitational lensing shows considerable promise. While beset by important issues involving number evolution and the determination of galaxy and cluster masses, ${ }^{22}$ counting galaxies and clusters of galaxies holds promise for probing the dark energy.

Two realistic goals for the next decade are determining $w$ to $5 \%$ and probing the time variation. Either has the potential to rule out a cosmological constant: For example, by measuring a significant time variation in $w$ or by pinning $w$ at $5 \sigma$ away from -1 . Such a development would be remarkable and far reaching.

After determining the equation-of-state of the dark energy, the next step is measuring its clustering properties. A cosmological constant is spatially constant; a rolling scalar field clusters slightly on very large scales.' 40 Measuring its clustering properties_will not be easy, but it provides an important, new window on dark energy. . $^{1}{ }^{\prime}$

We do live at a special time: There is still enough light in the Universe to illuminate the dark energy.

\section{Closing Remarks}

As a New Cosmology emerges, a new set of questions arises. Assuming the Universe inflated, what is the physics underlying inflation? What is the darkmatter particle? How was the baryon asymmetry produced? Why is the recipe for our Universe so complicated? What is the nature of the Dark Energy? Answering these questions will help us make sense of the New Cosmology as well as revealing deep connections between fundamental physics and cosmology. There may even be some big surprises - time variation of the constants or a new theory of gravity that eliminates the need for dark matter and dark energy.

There is an impressive program in place, with telescopes, accelerators, and laboratory experiments, both in space and on the ground: the Sloan Digital Sky Survey; the Hubble Space Telescope and the Chandra X-ray Observatory; a growing number of large ground-based telescopes; the Tevatron and B- 
factories in the US and Japan; specialized dark-matter detectors; gravity-wave detectors; a multitude of ground-based and balloon-borne CMB anisotropy experiments; the MAP satellite (which is already taking data) and the Planck satellite (to be launched in 2007). Still to come are: the LHC; a host of accelerator and nonaccelerator neutrino-oscillation and neutrino-mass experiments; the Next Generation Space Telescope; gravity-wave detectors in space; cluster surveys using x-rays and the Sunyaev - Zel'dovich effect. And in the planning: dedicated ground and space based wide-field telescopes to study dark energy, the next linear collider and on and on. Any one, or more likely several, of these experiments will produce major advances in our understanding of the Universe and the fundamental laws that govern it.

The progress we make over the two decades will determine how golden our age of cosmology is.

\section{Acknowledgments}

This work was supported by the DoE (at Chicago and Fermilab) and by the NASA (at Fermilab by grant NAG 5-7092).

\section{References}

1. See e.g., S. Weinberg, Gravitation and Cosmology (Wiley \& Sons, NY, 1972); or E.W. Kolb and M.S. Turner, The Early Universe (AddisonWesley, Redwood City, CA, 1990)

2. P. de Bernardis et al, Nature 404, 955 (2000); S. Hanany et al, Astrophys. J. 545, L5 (2000); C.B. Netterfield et al, iastro-ph/0104460; C. Pryke et al, istro-ph/ $/ 0104490$

3. D. Tytler et al, Physica Scripta T85, 12 (2000); J.M. O'Meara et al, Astrophys. J. 552, 718 (2001).

4. S. Burles et al, Phys. Rev. D 63, 063512 (2001); Astrophys. J. 552, L1 (2001).

5. M.S. Turner, 'astro-ph/0106035'

6. Y. Fukuda et al, Phys. Rev. Lett. 81, 1562 (1998).

7. R. Croft, W. Hu and R. Dave, Phys. Rev. Lett. 83, 1092 (1999).

8. L. Krauss and B. Chaboyer, astro-ph 2111597 ; I. Ferreres, A. Melchiorri, and J. Silk, MNRAS 327, L4 $\overline{7}(\overline{2} 001)$.

9. L. Knox, N. Christensen, and C. Skordis, astro-ph $0109232_{1}^{\prime}$

10. W.L. Freedman et al, Astrophys. J. 553, $47(2001)$.

11. J. Mather et al, Astrophys. J. 512, 511 (1999).

12. M.S. Turner, PASP 113, 653 (2001) (astro-ph/0102057)

13. See e.g., B. Sadoulet, Rev. Mod. Phys. $\overline{\mathbf{1}}, \overline{\mathrm{S}} 19 \overline{7}(\overline{9} \overline{9})$. 
14. See e.g., J. Sellwood and A. Kosowsky, astro-ph $/ 0 \overline{0} 0 \overline{90} \overline{7}$

15. D.N. Spergel and P.J. Steinhardt, Phys. Rev. Lett. 84, 3760 (2000).

16. M. Kaplinghat, L. Knox and M.S. Turner, Phys. Rev. Lett. 853335 (2000).

17. A. Kosowsky and M.S. Turner, Phys. Rev. D 52, R1739 (1995).

18. M.S. Turner, Phys. Rev. D 48, 5539 (1993); J.E. Lidsey et al, Rev. Mod. Phys. 69, 373 (1997).

19. S. Perlmutter et al, Astrophys. J. 517, 565 (1999)

20. A. Riess et al, Astron. J. 116, 1009 (1998)

21. A. Riess et al, Astrophys. J. 560,49 (2001).

22. M.S. Turner and A. Riess, astro-ph $/ 0106051$ (Astrophys. J., in press)

23. M.S. Turner and M. White, Phys. Rev. 5̄6, R4439 (1997)

24. S. Weinberg, Rev. Mod. Phys. 61, 1 (1989)

25. See e.g., http:/ /www.livingreviews.org/Ārticles/Volume4/2001-1carrolli

26. S. Thomas, hep-th/0010145!

27. E. Witten, hep-th/0106109:

28. T. Banks and $\bar{M}$. Dine, thep-th $/ 0106276$

29. S. Barr and D. Seckel, astro-ph/0106239!

30. M.S. Turner, Physica Scripta T85, $210(2000)$

31. A. Loeb, Astrophys. J. 499, L111 (1998)

32. See e.g., J. Newman and M. Davis, Astrophys. J. 534, L11 (2000); G.P. Holder et al, Astrophys. J. 553, 545 (2001); S. Podariu and B. Ratra, astro-ph $\angle 0106549^{\prime}$

33. See http://supernova.lbl.gov evlinder/sci.html

34. D. Huterer and M.S. Turner, Phys. Rev. D 64, 123527 (2001)

35. D. Huterer, 'astro-ph $\angle 106399_{1}^{\prime}$ (submitted to Phys. Rev. D)

36. S. Perlmutter, M.S. Turner, and M. White, Phys. Rev. Lett. 83, 670 (1999)

37. H. Martel, P. Shapiro, and S. Weinberg, Astrophys. J. 492, 29 (1998).

38. L. Krauss and M.S. Turner, Gen. Rel Grav. 31, 1453 (1999)

39. S. Carroll, Phys. Rev. Lett. 81, 3067 (1998)

40. K. Coble, S. Dodelson, and J. Frieman, Phys. Rev. D 55, 1851 (1997)

41. W. Hu and T. Okamoto, astro-ph 20111606 